[Feb., 1904.] TENTH ANNUAL MeEting OF THE SOCIETY. 221

\title{
THE TENTH ANNUAL MEETING OF THE AMERICAN MATHEMATICAL SOCIETY.
}

The Tenth Annual Meeting of the American Mathematical Societry was held in New York City on Monday and Tuesday, December 28-29, 1903. The following fortyeight members attended the four sessions :

Mr. Joseph Allen, Dr. Grace Andrews, Professor R. C. Archibald, Dr. C. L. Bouton, Professor Joseph Bowden, Professor E. W. Brown, Professor F. N. Cole, Miss E. B. Cowley, Dr. W. S. Dennett, Dr. Otto Dunkel, Professor F. C. Ferry, Dr. William Findlay, Professor B. F. Finkel, Professor T. S. Fiske, Dr. A. S. Gale, Dr. W. A. Granville, Dr. G. H. Hallett, Dr. J. G. Hardy, Professor J. Harkness, Dr. C. N. Haskins, Professor L. I. Hewes, Mr. E. A. Hook, Professor L. S. Hulburt, Dr. E. V. Huntington, Professor J. I. Hutchinson, Mr. L. L. Jackson, Dr. Edward Kasner, Professor C. J. Keyser, Mr. L. L. Locke, Dr. Emory McClintock, Professor James Maclay, Dr. Isabel Maddison, Mr. C. L. E. Moore, Professor F. Morley, Dr. L. I. Neikirk, Professor W. F. Osgood, Dr. I. E. Rabinovitch, Professor L. W. Reid, Dr. Arthur Schultze, Professor Charlotte A. Scott, Professor P. F. Smith, Dr. C. E. Stromquist, Professor H. W. Tyler, Mr. C. B. Upton, Professor E. B. Van Vleck, Professor J. M. Van Vleck, Dr. E. B. Wilson, Mr. J. E. Wright.

The President of the Society, Professor Thomas S. Fiske, occupied the chair, being relieved by Vice-President Professor W. F. Osgood. The Council announced the election of the following persons to membership in the Society: Mr. R. F. Deimel, Columbia University; Mr. C. S. Forbes, Columbia University ; Professor O. T. Geckeler, Georgia School of Technology ; Mr. E. A. Hook, Columbia University ; Mr. L. A. Martin, Jr., Stevens Institute of Technology; Miss Virginia Ragsdale, New York City ; Mr. S. E. Rasor, Ohio State University; Mr. A. E. Young, Purdue University; Mr. J. E. Wright, Bryn Mawr College. Six applications for membership in the Society were received.

Reports were presented by the Treasurer, the Auditing Committee, and the Librarian. These reports have already ap- 
peared in the Annual Register, published in January. During the past year the membership of the Society has shown an unusual increase, the net gain being 54, making the total membership 455 on January 1, 1904. The number of papers presented at the meetings of the Society in 1903 was 127 ; the total attendance at all the meetings was $222 ; 136$ members attended at least one meeting during the year. The library now contains over 1,300 bound volumes, an increase of some 300 since last year. The exchange list of the Bulluetis and the Transactions includes 154 journals. A catalogue of all journals in the library and of accessions other than journals received during the year is printed in the Annual Register. The Treasurer's report shows a balance of $\$ 2,573.07$ on hand, December 23,1903 . Of this balance $\$ 1,302.50$ is credited to the life-membership fund. The number of life members of the Society is now 30 .

At the annual election, which closed on Tuesday morning, the following officers and other members of the Council were chosen :

$\begin{array}{ll}\text { Vice-Presidents, } & \text { Professor Oskan Bolza, } \\ & \text { Professor J. M. VAN VLeck. } \\ \text { Secretary, } & \text { Professor F. N. Cole. } \\ \text { Treasurer, } & \text { Dr. W. S. DennetT. } \\ \text { Librarian, } & \text { Professor D. E. Smith. }\end{array}$

Committee of Publication,

Professor F. N. Cole,

Professor Alexander Ziwet,

Professor D. E. SMrTh.

Members of the Council to serve until December, 1906,

Professor Maxime Bôcher, Professor M. B. Porter, Professor Florian Cajori, Professor J. H. Tanner.

A committee consisting of the President and Professor Osgood was appointed to arrange for the publication in book form of the courses of lectures delivered by Professor E. B. Van Vleck, Professor H. S. White and Professor F. S. Woods before the Boston Colloquium, in September, 1903. A committee was also appointed to consider the question of the financial support of the Transactions after the expiration of the present university subscriptions. 
A pleasant social feature of the occasion was a dinner on Monday evening, which was attended by about thirty members of the Society.

The following papers were read at the annual meeting :

(1) Dr. E. V. Huntington : "A set of independent postulates for the algebra of logic (second paper)."

(2) Dr. J. G. Hun : "On certain invariants of two triangles."

(3) Dr. O. D. Kelloga : "Note on Cauchy's integral."

(4) Professor J. I. Hutchinson : "On certain automorphic functions."

(5) Professor W. F. Osaood : "On a gap in the usual presentation of Weierstrass's theory of functions."

(6) Dr. E. V. Huntington : "Third complete set of postulates for the theory of positive integers."

(7) Dr. E. V. Huntington: "Second complete set of postulates for the theory of magnitudes or positive real quantities."

(8) Dr. W. B. Fite : "On some properties of groups whose orders are powers of a prime."

(9) Professor E. J. Wilczynski : “ On ruled surfaces whose flecnode curve intersects every generator in two coincident points."

(10) Professor Virgil SNyder: "Complete enumeration of sextic scrolls having a rectilinear directrix."

(11) Professor F. Morley : "On the triplicity of 3-points in a plane."

(12) Mr. C. L. E. Moore : "Classification of surfaces of singularities in the quadratic spherical complex."

(13) Mr. L. D. Ames: "On the theorem of analysis situs relating to the division of the plane or of space by a closed curve or surface."

(14) Mr. W. B. Ford : "On the function defined by a Maclaurin series."

(15) Professor P. F. Sмiтh : "Linear transformation of a quadratic form into itself."

(16) Dr. E. B. WILSON : "Projective and metric geometry."

(17) Mr. C. H. Sisam : " On the depiction of the lines of a special linear complex on the points of space."

(18) Dr. EDWARD KASNER : "Investigations on isothermal systems."

Dr. Hun's paper was communicated to the Society through Dr. Kasner. Mr. Sisam was introduced by Professor Snyder. 
In the absence of the authors, Mr. Ames's paper was read by Professor Osgood, Dr. Fite's paper by Professor Hutchinson, and the papers of Dr. Hun, Dr. Kellogg, Professor Wilczynski, Professor Snyder, Mr. Ford, and Professor Smith were read by title. The papers of Dr. Kellogg, Professor Osgood, and Mr. Ames will appear in the Bulletis. Abstracts of the other papers follow below. The abstracts are numbered to correspond to the titles in the list above.

1. In the postulates of this paper Dr. Huntington expresses a necessary and sufficient condition that a class $K$, with a relation $<$, shall be equivalent to the class of logic classes with the relation of inclusion.*

1. $a<a$ whenever $a$ is in the class.

2. If $a<b$ and $b<a$, then $a=b$.

3. If $a<b$ and $b<c$, then $a<c$.

4. There is an element $z$ such that $z<a$ for every $a$.

5. There is an element $i$ such that $a<i$ for every $a$.

6. If $a \neq b$, there is an element $s$ such that 1) $a<s$; 2) $b<s ; 3)$ if $a<y$ and $b<y$ then $s<y$.

7. If $a \neq b$, there is an element $p$ such that 1) $p<a$; 2) $p<b$; 3) if $x<a$ and $x<b$ then $x<p$.

8. If the elements $z$ and $i$ in 4 and 5 exist and are unique, then for every element $a$ there is an element $a^{\prime}$ such that 1) if $x<a$ and $x<a^{\prime}$ then $\left.x<z ; 2\right)$ if $a<y$ and $a^{\prime}<y$, then $i<y$.

Definition $a \oplus b$. If $a \neq b, a \oplus b=s$ (see postulate 6 ); if $a=b, a \oplus a=a$.

Definition $a \odot b$. If $a \neq b, a \odot b=p$ (see postulate 7); if $a=b, a \odot a=a$.

9. If the element $z$ in 4 exists uniquely, and if the elements $s$ and $p$ in 6 and 7 exist and are uniquely determined by $a$ and $b$; and if, further, $b \odot c=z$; then $a \odot(b \oplus c)=(a \odot b) \oplus(a \odot c)$.

These nine postulates are shown to be independent.

2. With two plane curves, one of the $n$th order $\alpha_{x}^{n}=0$ and the other of the $n$th class $a_{\xi}^{n}=0$, there is associated the connex $\alpha_{y} a_{\eta} \alpha_{a}^{n-1}=0$. Some of the invariants of the two curves may be expressed by means of the invariants of this connex. In Dr. Hun's paper the curves considered are a 3-line and a 3point. A few of the simpler invariant relations of the two triangles are expressed in terms of the invariants of the connex.

* Cf. Schroeder, Algebra der Logik, and papers by C. S. Peirce. 
From the forms of these relations the truth of certain wellknown theorems may be seen immediately, e.g., that two conics apolar to a conic are circumscribed to a conic. By this method it is also shown that the relation between the fixed triangle of a normal collineation and a Pasch triangle belonging to that collineation is a mutual one. Toward the end of the article some attention is devoted to a conjugate 4-point of a 3-line, and to the collineations leaving fixed three points of the 4point. The paper will appear in the January number of the Transactions.

4. The paper by Professor Hutchinson discusses the functions belonging to the group whose fundamental region is a triangle having angles $\pi / 3, \pi / 6, \pi / 6$. The existence of certain multiplier functions is established, these having the character of entire functions and vanishing (of the first order) only at the fixed points for elliptic substitutions. In terms of these simplèst functions, all other functions of the group are expressed.

6. Dr. Huntington shows that a class $K$ with a rule of combination $\oplus$ will be equivalent to the class of positive integers with the rule of combination + , when and only when the following eight postulates are satisfied :

I-IV. The same as postulates I-IV in the paper below, (number 7).

$\mathrm{V}^{\prime}$. If there is an element $E$ having the property described in postulates $\mathrm{VI}^{\prime}$, then for every element $a$ there is a natural number $m$ such that $a \leqq m E$.

[Here $a \leqq b$ has the same significance as in $\mathrm{V}$, in the paper cited, while $m E$ indicates the element defined by the following recursion formulæ : $1 E=E, 2 E=1 E \oplus E, 3 E=2 E \oplus E, \cdots$, $(m+1) E=m E \oplus E$.

$\mathrm{VI}^{\prime}$. There is an element $E$ such that $x \oplus y \neq E$ whenever $x \neq E$ and $y \neq E$. cited.

VII, VIII. The same as postulates VII, VIII in the paper

The postulates of this third set, like those of the two earlier sets (of six and of four postulates respectively: Transactions, volume 3, page 280), are shown to be consistent, sufficient and independent.

7. Dr. Huntington shows that a class $K$ with a rule of combination $\oplus$ will be equivalent to the class of positive real quanti- 
ties with the rule of combination + , when and only when the following eight postulates are satisfied :

I. $a \oplus b$ belongs to the class whenever $a$ and $b$ belong to the class.

II. If $a \neq b$, then $a \oplus b \neq a$.

III. $(a \oplus b) \oplus c=a \oplus(b \oplus c)$, whenever $a \oplus b, b \oplus c$, etc., belong to the class.

IV. If $a \neq b$, then there is either an element $x$ such that $a=b \oplus x$, or an element $y$ such that $a \oplus y=b$.

$\mathrm{V}$. (Let the notation $a<b$ indicate that an element $y$ exists such that $a \oplus y=b$; and let $a \leqq b$ indicate : $a<b$ or $a=b$.)

If $S$ is an infinite sequence of elements $\left(\alpha_{k_{k}}\right)$, such that

$$
a_{k}<a_{k+1}, a_{k}<c \quad(k=1,2,3, \cdots)
$$

(where $c$ is some fixed element), then there is an element $A$ having the following two properties:

1) $a_{k} \leqq A$ whenever $a_{b}$ belongs to $S$.

2) If $B<A$, then there is at least one element of $S$, say $a_{r}$, for which $B<a_{r}$.

VI. For every element $a$ there are two elements $x$ and $y$ such that $x \oplus y=a$.

VII. $a \oplus b=b \oplus a$ whenever $a, b, a \oplus b$, and $b \oplus a$ belong to the class.

VIII. There are two elements $x$ and $y$ in the class, such that $x \neq y$.

The postulates of this new set, like those of the earlier set of six postulates (Transactions, volume 3, page 264) are shown to be consistent, sufficient, and independent.

8. Dr. Fite's paper concerns itself with the generalization of some theorems proved by him in the Transactions and in the Bulletin. It considers the invariance of certain powers of operators of groups whose orders are powers of a prime, and also the possibility of certain groups occurring as groups of cogredient isomorphisms.

9. Professor Wilczynski gave a complete geometric interpretation for the covariant $C_{3}$ in the case where the flecnode curve intersects every generator in two coincident points, the interpretation given for the general case not being applicable. $\mathrm{He}$ further showed how to associate, with every generator of the ruled surface, a certain conic, the derivative conic. This 
gives rise to a surface, associated with the ruled surface, upon which are situated. a single infinity of conics. A number of theorems were proved in regard to the derivative conic, and also in regard to the developable surface enveloped by the plane of the derivative conic.

10. By employing the same methods as those published in the American Journal, volume 25, and the additional property that a bitangent from a point on the directrix to a plane section of a scroll shows the presence of a double point on the nodal curve, Professor Snyder has proved the existence and obtained the equations of over one hundred forms of sextic scrolls having a rectilinear directrix. The list is believed to be complete.

11. If three points are severally on and collectively apolar with a cubic curve in a plane, four linear conditions are imposed on the cubic. Two such 3-points in general impose eight conditions and determine a pencil of cubics, which intersect also in a third 3-point.

In Professor Morley's paper it was shown that the three 3 -points are mutually related, so that the 3-points of a plane, projectively considered are in a relation like an involution, say a triplicity. In proof, two cubics of the pencil are taken, one the resultant $(a b x)^{3}$ of the 3-points $(a \xi)^{3}$ and $(b \xi)^{3}$, the other a rational cubic, along which a parameter $\kappa$ is spread. The three triads of parameters where the cubics meet are shown to be mutually related.

For proper 3-points, that is, not on a line, there is the exceptional or 'neutral' case when the resultant explodes; that is, the two given 3-points appear apolar from every point on the plane. When two such 3-points are on a cubic, they are apolar with it, so that the eight conditions cease to be independent.

12. The general surface of singularities in the spherical quadratic complex has been studied by Professor P. F. Smith in the Transactions, volume 1, page 381. By using the same methods and supplementing them with the types of cyclides as given by Loria, Mr. Moore has completed the classification of all the types, including those which are annular surfaces, scrolls, developables, cyclides and minimum lines.

14. The results contained in Mr. Ford's paper relate to the function of the complex variable $z$ defined by the series 


$$
a(0)+a(1) z+a(2) z^{2}+\cdots+a(k) z^{k}+\cdots,
$$

both in the case where the radius of convergence of the series is not equal to zero and where equal to zero. In the first of these cases a theorem is obtained by which the function defined by (1) may be studied when the same function could not be studied by the direct application of any theorem relating to this subject which has appeared heretofore, including those of Le Roy and Lindelöf and the theorem established a short time since by the author in the Journal de Mathématiques. In each of these former theorems, in fact, it is required to know certain facts concerning the behavior of the function $a(k)$ in the neighborhood of the point $k=\infty$. If we have at our command, however, only the means of studying $a(k)$ for finite values of $k$ (as is in general the case), the facts just cited become difficult in many cases to determine. But by means of the theorem which Mr. Ford establishes, one may avoid such difficulty, at least in very many cases, since by it the discussion of the function $a(k)$ is referred to that of two other functions $b(k)$ and $c\left(w_{1}, w_{2}, w_{3}, \cdots, u_{q}\right)$, each of which for a given series (1) may usually be chosen in various ways, at least one of which will be such that the conditions demanded will be satisfied. In the second of the cases cited above a theorem is obtained by which one may study the function defined formally by a divergent series (1).

15. The point of view adopted in Professor Smith's paper was announced in a preliminary communication made to the Society in December, 1901, entitled: "On the Resolution of Orthogonal Transformations into Involutory Transformations." The present paper completes the discussion of the general question of linear transformation of an invariant quadratic form, obtaining in explicit form all such transformations, discusses the resolution into involutory transformations and closes with an application to the case of six variables, completing the theorem given by Dr. Wilson in the Transactions concerning the resolution of a collineation into three skew reflections.

16. The correlation between projective and metric parabolic geometry seems unsatisfactory : for first, the method of using the absolute depends on imaginaries and on a limiting process, and secondly, the procedure employed in most books on pure geometry avoids the question entirely and merely states metric 
results in projective language. In this note, which has been offered to the Annals of Mathematics, Dr. Wilson shows how to pass from the general projective geometry to metric parabolic geometry by means of a fundamental unit conic and Von Staudt's theory of involutions.

17. Mr. Sisam discussed a depiction of the lines of a special linear complex on the points of space, as developed by Anders Wiman, of Lund, and applied it to the study of quintic scrolls. The polar planes of an arbitrary point with respect to a pencil of quadrics intersecting in two conics form a pencil whose axis cuts the line of intersection of the planes of the conics. By letting this axis correspond to the given point, we have the depiction required. A scroll belonging to the complex depicts into a curve. By studying the curves corresponding to quintic scrolls, thirty-eight types were found which have a rectilinear directrix.

18. Dr. Kasner's paper is a contribution to the geometry of ordinary differential equations, that is, of systems of curves. Three topics in particular are discussed: $1^{\circ}$ The determination of the linear differential equations which represent isothermal systems. $2^{\circ}$ The derivation of new isothermal systems from known systems depending on the consideration of slope systems (the slope curves of a given system $y^{\prime}=f(x, y)$ are represented by $f(x, y)=$ const.). $\quad 3^{\circ}$ The characterization of an isothermal system in terms of the isogonal trajectories. The result obtained here applies to systems on a surface as well as in a plane, and is as follows: The $\infty^{2}$ isogonal trajectories of a given system of $\infty^{1}$ curves constitute a linear two-parameter system when, and only when, the given system is isothermal. Incidentally certain general theorems on isogonal trajectories are obtained.

Columbia University.

F. N. Cole. 